\title{
Pre-vaccination allergy testing with COVID-19 mRNA vaccines predicts tolerance
}

\author{
Florian Stehlin ${ }^{1}$, Rima Mahdi-Aljedani ${ }^{1}$, Loris Canton ${ }^{1}$, Véronique \\ Monzambani-Banderet ${ }^{1}$, Alix Miauton ${ }^{2}$, Cedric Girard ${ }^{1}$, Kevin Kammermann ${ }^{3}$, Sylvain \\ Meylan ${ }^{1}$, Camillo Ribi ${ }^{1}$, Thomas Harr ${ }^{4}$, Daniel Yerly ${ }^{3}$, and Yannick Muller ${ }^{1}$ \\ ${ }^{1}$ Centre Hospitalier Universitaire Vaudois \\ ${ }^{2}$ Policlinique Médecine Tropicale Voyages et Vaccinations Unisanté Lausanne \\ ${ }^{3}$ Adverse Drug Reactions - Analysis \& Consulting (ADR-AC) GmbH Bern Switzerland \\ ${ }^{4}$ Hopitaux Universitaires Geneve
}

September 28, 2021

\begin{abstract}
Background: The newly developed mRNA-based COVID-19 vaccines can provoke anaphylaxis, possibly induced by polyethylene glycol (PEG) contained in the vaccine. The management of persons with a history of PEG allergy, or with an allergic-like reaction after the first dose remains to be defined. Methods: We studied two cohorts of individuals: one pre-vaccination, the second post-vaccination. Skin testing was performed with COVID-19 mRNA vaccines. Upon negative skin test, a two-step (10\%-90\%) vaccination protocol was performed. Positive skin tests were confirmed with basophil activation tests (BAT). Vaccine-sensitized patients were offered a five-step induction protocol. Results: We identified 187 patients with high-risk profiles for developing anaphylaxis. In parallel, among 385'926 doses of vaccine, 87 allergic-like reactions were reported to our division for further investigations: 18/87 (21\%) were consistent with anaphylaxis, 78/87 (90\%) were female, and 47/87 (54\%) received the BNT162b2 mRNA vaccine. Vaccine skin tests were negative in $96 \%$ and $76 \%$ in the pre- and post-vaccination cohorts, respectively. A twostep vaccination was tolerated in 232/236 (98\%) of individuals with negative tests. Four individuals experienced acute asthma exacerbation during the two-step challenge. Vaccine-positive skin tests were consistently confirmed by BAT; CD63 and CD203c expression was selectively inhibited with ibrutinib, suggesting an IgE-dependent mechanism. Finally, 13 sensitized patients were successfully vaccinated with a five-step vaccination protocol. Conclusion: A two-step 10\%-90\%-vaccination protocol can be safely administered upon negative skin testing. Yet, it should be delayed in individuals with poorly controlled asthma. Importantly, mRNA vaccine sensitized individuals may receive a five-step vaccination protocol.
\end{abstract}

\section{Pre-vaccination allergy testing with COVID-19 mRNA vaccines predicts tolerance}

Florian Stehlin (1), Rima Mahdi-Aljedani* (1), Loris Canton* (1), Véronique Monzambani-Banderet (1), Alix Miauton (2), Cedric Girard (3), Kevin Kammermann (4), Sylvain Meylan (5), Camillo Ribi (1), Thomas Harr (6), Daniel Yerly (4), Yannick D. Muller (1)

(1) Division of Immunology and Allergy, University Hospital of Lausanne, Switzerland

(2) Policlinique Médecine Tropicale, Voyages et Vaccinations, Unisanté, Lausanne

(3) Pharmacy, University Hospital of Lausanne, Switzerland

(4) Adverse Drug Reactions - Analysis \& Consulting (ADR-AC) GmbH, Bern, Switzerland

(5) Infectious Diseases Service, University Hospital Lausanne and University of Lausanne, 
Switzerland

(6) Division of Immunology and Allergy, University Hospital of Geneva, Switzerland

*Contributed equally

Correspondence:

Yannick Daniel Muller - MD-PhD - Assistant Professor

Service d'immunologie et d'allergie, Département de médecine, BH010-511 Rue du Bugnon 46, CH-1011 Lausanne Mob: +41(0)795569438 ; Email :Yannick.muller@chuv.ch.

Short title: Allergy and COVID-19 mRNA vaccines

Keywords : SARS-Cov2, Allergy, Anaphylaxis, COVID-19, Vaccine, excipient, macrogol.

Background:

The newly developed mRNA-based COVID-19 vaccines can provoke anaphylaxis, possibly induced by polyethylene glycol (PEG) contained in the vaccine. The management of persons with a history of PEG allergy, or with an allergic-like reaction after the first dose remains to be defined.

\section{Methods:}

We studied two cohorts of individuals: one pre-vaccination, the second post-vaccination. Skin testing was performed with COVID-19 mRNA vaccines. Upon negative skin test, a two-step (10\%-90\%) vaccination protocol was performed. Positive skin tests were confirmed with basophil activation tests (BAT). Vaccinesensitized patients were offered a five-step induction protocol.

\section{Results:}

We identified 187 patients with high-risk profiles for developing anaphylaxis. In parallel, among 385'926 doses of vaccine, 87 allergic-like reactions were reported to our division for further investigations: 18/87 (21\%) were consistent with anaphylaxis, 78/87 (90\%) were female, and 47/87 (54\%) received the BNT162b2 mRNA vaccine. Vaccine skin tests were negative in $96 \%$ and $76 \%$ in the pre- and post-vaccination cohorts, respectively. A two-step vaccination was tolerated in 232/236 (98\%) of individuals with negative tests. Four individuals experienced acute asthma exacerbation during the two-step challenge. Vaccine-positive skin tests were consistently confirmed by BAT; CD63 and CD203c expression was selectively inhibited with ibrutinib, suggesting an IgE-dependent mechanism. Finally, 13 sensitized patients were successfully vaccinated with a five-step vaccination protocol.

\section{Conclusion:}

A two-step 10\%-90\%-vaccination protocol can be safely administered upon negative skin testing. Yet, it should be delayed in individuals with poorly controlled asthma. Importantly, mRNA vaccine sensitized individuals may receive a five-step vaccination protocol.

\section{Introduction}

The newly developed anti-SARS-Cov2 COVID-19 mRNA-vaccines (BNT162b2 mRNA from Pfizer-BioNTech and mRNA-1273 from Moderna) represent a potential exit strategy from the ongoing severe COVID-19 pandemic, but are associated with rare cases of anaphylaxis[1-3]. The first severe cases of anaphylaxis were rapidly reported after the start of the worldwide vaccination campaign, and led the US and European centers for disease prevention and control to recommend a 15-minute surveillance post-vaccination, which should be prolonged to 30 minutes in any patient with a history of severe anaphylaxis. The vaccination is currently contraindicated for persons with an allergic reaction to the COVID-19 vaccine or known allergies to any of its components. This includes polyethylene glycol (PEG), or macrogols, and polysorbate, the latter being known to cross-react with PEG, as it is constituted of PEG molecules [4]. 
In the US, the incidence of allergic reactions to mRNA vaccines was initially estimated 10 times higher than for other virus-based vaccines [2, 3]. More recently, based on a metanalysis regrouping 41'000'000 vaccinations and a more recent study, the incidence of SARS-Cov2 vaccine anaphylaxis is estimated between 4.7-7.9 cases per million vaccinations [5-7]. Expert opinions and a number of small case reports suggest that people with immediate allergic reactions to mRNA vaccines are likely sensitized to PEG [7-10]. Urgent research has been recommended by a large panel of international experts to define the utility of skin testing to identify risk for allergic reactions to the vaccines or their excipients in allergic individuals [7].

For evident safety reasons, in our center we rapidly established a diagnostic routine protocol, consisting of vaccine drug skin testing, followed by a two-step vaccination protocol (10\%-90\%). This diagnostic routine protocol included two categories of individuals: The first one regrouped individual before vaccination, with prior history of anaphylaxis to any injectable drug (intravenous, subcutaneous, or intramuscular) that contained PEG, polysorbate or trometamol. Were also included patients with a documented allergy to PEG and/or Polysorbate. In selected cases, where the presence/absence of those excipients could not be established, patients were also invited to get tested before vaccination. The second category regrouped persons after vaccination that showed immediate-like allergic reactions, following the first or second dose of vaccine.

Herein, we report the results of skin tests, basophil activation test (BAT) and clinical tolerability of a twoand five-step vaccination protocol in patients respectively with negative and inconclusive/positive skin tests. Vaccine hypersensitivity was found in $4 \%$ of the pre-vaccination cohort and in $24 \%$ of the post-vaccination cohort. Importantly, negative skin tests predicted vaccine tolerance in $98 \%(232 / 236)$ of the patients. A five-step induction protocol was successfully administered in thirteen vaccine-sensitized patients.

\section{Methods}

Study approval

The study was approved by the local Ethics Committee of the canton of Vaud, Switzerland (BASEC number 2021-00735). Written informed consent was obtained from all participants.

Pre-vaccination cohort

The canton of Vaud in Switzerland has over 800'000 inhabitants. To get an appointment for the vaccination, every person went through a standardized evaluation, either online (https://coronavax.unisante.ch/evaluation) or via a hotline. During the evaluation, every person had to respond to the following questions:

1. Do you have allergies?

2. Do you have documented allergy to any of the vaccine components* (*Polyethylene-Glycol (PEG, macrogol), polysorbate, tromethamine (TRIS, trometamol), or to PEG-derived laxatives?

3. Did you previously suffer from severe allergic reaction* to infused or injectable drugs? (*history of anaphylactic shock, that required the prescription of adrenaline, a life-threatening allergic reaction)

4. Did you previously suffer from a severe allergic reaction of other origins* (*oral medication, food allergy, latex, pollen, animals, dust mites, insect venom)?

If the answer to question 1 was no, questions 2-4 were not asked. Persons with a yes answer to questions 2 and/or 3 were asked to get medical advice to assess their eligibility for the vaccination, either by contacting their general practitioner, or by competing an online form which was sent to our allergy division (see results section). Persons who responded yes to question 4 only were eligible for a vaccination with 30-minutes surveillance. The standardized workup consisted of skin tests with one of the two anti-SARS-Cov2 mRNA vaccines. Skin prick tests (SPT) with histamine and $\mathrm{NaCl} 0.9 \%$ were used as positive and negative controls, respectively. If skin tests returned negative, patients were offered on the same day a two-step (10\%-90\%) vaccination protocol, with 30-minutes of surveillance after each injection. If skin tests returned positive, a complimentary workup was done, including whenever possible both BNT162b2 mRNA and mRNA-1273 vaccines (SPT 1:1, intra-dermal-reaction (IDR) dilution 1/100), polysorbate-80 1\% (dilution 1/10), PEG-2000 1\% (dilution $1 / 100$ ), and trometamol $1 \%$ (dilution 1/100), the latter being an excipient of the mRNA-1273 vaccine. IDR 
with $\mathrm{NaCl}$ 0.9\% was used as a negative control. According to the European Network of Drug Allergy (ENDA) guidelines, a skin test was considered positive in case of a papule of $3 \mathrm{~mm}$ or more, in comparison to the steady-state with erythema at 20 minutes. In case of a papule evolving from the steady-state, but of less than $3 \mathrm{~mm}$, the test was considered inconclusive (suspicious).

\section{Post-vaccination cohort}

In this cohort, we included all patients with a history of allergic-like reactions after the first or second injection with mRNA vaccine against COVID-19. Individuals with an allergic reaction, but not meeting anaphylaxis criteria as per European Academy of Allergy and Clinical Immunology (EAACI) definition [11-13], received an appointment in our division of allergy for a standardized workup, consisting of skin testing with one of the two mRNA vaccines as described above. If skin tests returned positive, the same complementary skin tests described above were performed. Individuals experiencing an anaphylactic reaction (as per EAACI definition) after the first mRNA vaccine dose, received an appointment for skin testing only, with at least one of the two mRNA vaccines, polysorbate-80, PEG-2000, and trometamol. In most of the cases, upon positive or suspicious skin test results, a BAT was performed to confirm the skin test results. In vaccine-hesitant patients with negative skin tests results, we also performed BAT.

\section{Basophil activation tests}

Peripheral blood was obtained from individuals from the pre- or post-vaccination cohorts. Briefly, $100 \mu \mathrm{L}$ of peripheral blood per test condition was incubated with or without IL-3 $(1 \mathrm{ng} / \mathrm{mL})$ with different concentration of vaccines $(1 \%, 0.1 \%, 0.01 \%)$ or Polysorbate- $80(10 \mu \mathrm{g} / \mathrm{ml}, 2 \mu \mathrm{g} / \mathrm{ml}, 0.4 \mu \mathrm{g} / \mathrm{ml})$ for $30 \mathrm{~min}$ at $37^{\circ} \mathrm{C}$, along with anti-CCR3 PE and anti-CD63 FITC, anti-CD203 APC antibodies (Biolegend, San Diego, CA). Control conditions included a medium-only negative control, a positive control, involving the crosslinking of the highaffinity Fc epsilon receptor (anti-IgE) (Beckmann-Coulter, Brea, CA), and a positive control independent of FcERI signaling, $N$-formylmethionyl-leucyl-phenylalanine (fMLP) (Sigma-Aldrich, St. Louis, Missouri) [13]. Flow cytometry data were collected on NovoCyte (Agilent Technologies, Santa Clara, CA).

\section{Statistics}

Multiple unpaired t-test was performed using GraphPad Prism version 9 for Macintosh (GraphPad Software, San Diego, California USA). Mean and standard deviations are shown. Fisher's exact tests were performed using IBM SPSS Statistics for Macintosh, Version 27.0.

\section{Results}

\section{Pre-vaccination cohort}

During the first 6 months of the vaccination campaign, we evaluated 2'477 online forms from vaccine candidates with allergies and called in 200 persons with a high-risk profile for vaccine anaphylaxis for work-up at our outpatient clinic. Thirteen patients did not provide informed consent for this study and were therefore excluded from the analysis. Mean age was 62.7-year-old, 145/187 (78\%) were female, and 150/183 (82\%) had a prior history of anaphylaxis (Supplementary Table 1 ). One of the first cases with positive skin tests was a 55-year-old patient with prior history of generalized pruritus, urticaria, dyspnea, and dysphonia minutes after starting a preparation of macrogol 3350 for a colonoscopy in 2020. The patient underwent SPT and IDT skin testing: SPT was inconclusive for the mRNA-1273 vaccine, but otherwise negative. IDT was strongly positive for the BNT162b2 mRNA and mRNA-1273 vaccines, and polysorbate- 80 and negative for PEG-2000 and trometamol (Figure 1A ). We found six other patients with positive skin tests for mRNA vaccines. Interestingly, $5 / 7$ had also positive IDR for polysorbate-80, but none of them was positive for PEG-2000 or trometamol. In addition, none of them had unclear or multiple unidentified drug allergies/anaphylaxis (Figure 1B, Supplementary Table 2 ). Patients with negative skin tests were offered a 2-step (10\%-90\%) vaccination protocol, which was accepted by $178 / 180$ patients. Vaccine tolerance was $99.4 \%$. One patient developed an asthma attack which required 12-hour monitoring at the emergency department.

Post-vaccination cohort 
In parallel, our division of allergy was in charge of evaluating allergic-like reactions after the first or second dose. As of June 16th 2021, 385'926 doses of mRNA-based vaccine were injected in the canton Vaud. Of the 104'561 vaccine doses administered at the University Hospital of Lausanne (CHUV), 93'890 (90\%) were the BNT162b2 mRNA vaccine, and 10'671 (10\%) the mRNA-1273 vaccine. Thirty-five allergic-like reactions (3.3 reactions $/ 10$ '000 doses) were reported at the vaccine center of CHUV, and overall, 93 from all vaccine centers in the canton Vaud (2.4 reactions/10'000 doses) (Figure 1B ).

Among the 93 evaluated patients, 87 provided informed consent for the study. Mean age was 48.4-year-old, and $90 \%$ were women. Three of $87(3 \%)$ had a history of allergy to drugs sharing excipients with the mRNA vaccines (Supplementary Table 2 ). Significantly fewer individuals had negative skin tests compared to the pre-vaccination cohort ( $76 \%$ versus $96 \%$ respectively). Importantly, over $95 \%$ of individuals with negative skin tests tolerated a two-step rechallenge protocol (Figure 1B ). Three individuals, developed an asthma attack, one after the $10 \%$ step and two after the $90 \%$ step. All required a monitoring at the emergency department.

Workup yielded positive skin-tests to the vaccine in $9 / 86(10 \%)$ and remained inconclusive in 12/86 (14\%) of patients. Skin tests with PEG-2000 were negative in 19/21 cases and inconclusive in $2 / 21$. Skin tests with trometamol were negative in all 21 patients. Finally, among individuals with inconclusive/positive skin tests for mRNA vaccines, (10/21) 48\% had inconclusive/positive skin tests for polysorbate-80. Positive skin tests were significantly associated with younger age and the mRNA-1273 vaccine (Table 1 ). However, neither the symptoms, timing of symptoms, criteria for anaphylaxis, Brighton Score (level 0-3 defining the certainty of anaphylaxis), nor the Ring and Messmer Severity Scale (1-4 grading system of clinical anaphylaxis severity) predicted skin test positivity to the mRNA vaccine (Figure 1B ).

Positive vaccine skin tests correlate with BAT results

To further investigate the inconclusive and/or positive skin tests with either mRNA vaccine, we performed BAT in 24 individuals from the pre- and post-vaccination cohorts (Figure 2A-C ). As controls, we included 14 individuals, among which 4 were healthy without history of allergy (skin tests not available), one had dermographism, two had inconclusive skin tests for polysorbate- 80 but were negative for the vaccine, and 7 were vaccine-hesitant.

BATs were performed at the ADR-AC Laboratory in Bern, Switzerland. Interpretation was made independently of the skin test results. Strikingly, BAT results consistently confirmed inconclusive and/or positive skin tests with the vaccine, while individuals with negative skin test results had negative BAT results (Figure 2D ). The results also showed cross-reactivity between the mRNA-1273 and BNT162b2 mRNA vaccines, which we also observed with skin testing. CD203c appeared to be the best marker to discriminate between positive and negative BAT results across all different concentrations of vaccine tested (Figure 2A-C ). Finally, while no significant difference was found for polysorbate- 80 positivity between vaccine positive and negative BAT, we observed a few outliers in vaccine-sensitized individuals only.

Vaccine-induced basophil activation is IgE-dependent

Ibrutinib is a Bruton's tyrosine kinase inhibitor which has been shown to target IgE-dependent secretion of histamine in basophils [14]. To investigate whether vaccine-induced upregulation of CD63 and CD203c is IgEdependent, we performed a BAT in five vaccine-sensitized individuals with and without ibrutinib (Figure 3 ). As previously demonstrated [14], ibrutinib selectively inhibited CD63 upregulation upon basophil simulation with anti-Fce receptor I antibodies, but not with the bacterial peptide fMLP. Yet, we also observed a partial but significant inhibition of CD203c upon fMLP stimulation with ibrutinib, suggesting that this drug also has some IgE-independent activity. Importantly, in vaccine-stimulated conditions, we found a significant downregulation of basophils' CD63 and CD203c expression in the presence of ibrutinib, suggesting that the upregulation of those markers is, at least partially, IgE-dependent.

Individuals with positive skin tests may be successfully vaccinated with a 5-step induction protocol

Considering that the mechanisms of basophil activation with mRNA vaccines are possibly IgE-dependent, 
we decided to establish a 5-step induction protocol for vaccine sensitized individuals, using an algorithm established by the American Academy of Pediatrics, which we adapted for the BNT162b2 mRNA and the mRNA-1273 vaccines [15] (Supplementary Table 3). This decision was further motivated by a single sensitized patient that we decided to rechallenge with a two-step vaccination protocol, associated with urticaria recurrence (Figure 1B ).

As of September 2021, 14 female patients underwent the 5-step vaccination procedure, of which 4 from the pre-vaccination cohort and 10 from the post-vaccination cohort. Except for one patient who had an acute asthma exacerbation during the 2-step vaccination procedure despite negative skin testing, all individuals had inconclusive and/or positive skin tests that were confirmed by BAT when possible (Table 2 ). Importantly, all 14 patients (including 13 vaccine-sensitized individuals) could be successfully vaccinated using the 5step protocol. Adverse events during the procedure were observed in three cases: two cases experienced a mild asthmatic exacerbation, which responded well to beta-mimetics only and another patient developed a localized urticaria at the injection site. These results suggest that a five step-vaccination protocol can be proposed to vaccine-sensitized patients, provided that there is an adequate setting for surveillance and treatment of anaphylaxis.

\section{Discussion}

This is currently the largest study investigating vaccine sensitization in two cohorts of patients, either before vaccination individuals with a high risk for anaphylaxis, or after vaccination in individuals with allergic-like reactions. We showed that negative skin testing predicts vaccine tolerance independently of the severity of the initial reaction. These data emphasis the usefulness of skin testing to decide whether patients are eligible for the primary vaccination or a booster dose. Importantly we demonstrate that CD63 and CD203c upregulation in basophils was at least partially IgE-dependent. Finally, we showed that most sensitized patients tolerate a 5 -step induction protocol with either mRNA vaccine.

The incidence and/or prevalence of allergic-like reactions for mRNA-based vaccines is intensely debated. Multiple definitions of anaphylaxis, data collected from national agencies/registries versus prospective clinical studies, but also efforts made to determine the validity and causality of all allergic-like reactions hampered these estimations $[7,16]$. Ongoing studies are trying to prospectively evaluate the proportions of systemic allergic reactions to the BNT162b2 mRNA and mRNA-1273 vaccines (NCT04761822). Yet, based on our experience, we estimated that there are 2.4-3.3 suspect allergic-like reactions per 10'000 doses. These numbers are comparable to the observational study performed at the Mass General Brigham hospital that concluded on an estimated rate of severe allergic reaction or anaphylaxis of 2.47 per 10000 vaccinations [17].

In our study, half of the reactions started 60 minutes or more after vaccination and only $21 \%$ met the criteria anaphylaxis (EAACI). Very importantly, we did not observe any fatalities, nor had to admit any patient to the intensive care unit because of anaphylaxis. Several reasons may account for this observation: First, the 15-30 min clinical surveillance helps to rapidly identify and treat allergic reactions. Secondly, we established an online screening form to identify patients with severe allergic reactions to injectable drugs containing PEG/polysorbate before vaccination. Third, skin testing identifies mRNA vaccine-sensitized patients which can then be excluded from a standard (re)vaccination protocol.

Overall, we found that IDR tests with mRNA-vaccines have a sensitivity of $>98 \%$. The sensitivity of SPT was however very poor, as none of them returned positive, suggesting that they may be omitted in the context of this pandemic to gain time in the allergy workup. In 4 cases out of 236 vaccinations, we had an asthmatic reaction despite negative skin tests. While further investigations are needed to define the mechanisms involved in these reactions, we strongly recommend that asthmatic patients with poor asthma control postpone their vaccination until their asthma is properly controlled. Based on the results of this study, it seems reasonable to restrict mRNA vaccine skin tests before vaccination to patients with documented or highly-suspected allergy to PEG and/or Polysorbate and to consider them only in vaccinehesitant individuals with unspecific history of drug-anaphylaxis or multiple drug allergies to increase global coverage of vaccination. 
PEG is the suspected culprit for mRNA-vaccine allergic-like reactions $[1,7,9]$ as it is known to provoke IgE-mediated anaphylaxis[4, 10, 18]. Yet, there are no clear recommendations for testing PEG in patients with either suspected SARS-CoV-2 mRNA allergy, or allergy to an injectable drug containing PEG. A recent systematic review performed by a multidisciplinary group of international experts identified 21 studies on skin testing with PEG and/or polysorbate- 80 [4, 7, 10, 19-26]. They concluded on a general sensitivity of $58.8 \%$ and specificity of $99.5 \%[7]$.

Yet, in our two cohorts of patients, PEG-2000 skin testing (SPT 1\% and IDR 0.01\%) was negative in the majority of cases and inconclusive in very few. Several reasons may explain these findings: first, we intentionally used a low concentration of PEG-2000, as it was reported that IDT with a high PEG concentration can provoke anaphylaxis [10]. Second, most studies on PEG-allergic patients have used PEG of different molecular weights, ranging from 200 to 20 '000 [4, 7, 10, 19-26]. Thus, PEG-2000 has so far never been validated for diagnosing mRNA vaccine hypersensitivity $[8,27]$. Thirdly, several groups used surrogate drugs such as Movicol (PEG 3350) or methylprednisolone acetate (PEG 3350) to diagnose PEG allergy, instead of purified PEG, which are have not included in this study [4].

The tridimensional conformation of PEG may play an important role in the binding of anti-PEG IgE or, as suggested by some, of complement-dependent anti-PEG-IgM/IgG [28, 29]. In conclusion, we recommend against skin testing with PEG-2000 and rather privilege skin testing with mRNA vaccine and polysorbate80. The sensitivity of polysorbate- 80 skin testing to diagnose vaccine hypersensitivity has however yet to be validated, since only $48-86 \%$ (19-71\% if considering positive skin tests only) in respectively the post and pre-vaccination cohorts had cross-reactive skin tests with mRNA vaccines. These results, however, strongly suggest that there is a cross-reactivity between mRNA vaccines and polysorbate- 80 hypersensitivity.

For the first time, we showed that the majority of vaccine-sensitized patients tolerate a 5-step mRNA vaccination protocol without adverse events. The decision about the booster vaccination in allergic patients' needs to be made individually, balancing the risk and benefits. However, since in Switzerland there are no alternative anti-SARS-Cov2 vaccines without PEG and/or polysorbate and the booster dose is associated with a substantial gain in antibody neutralizing capacities [30,31], we advised our patients to consider this induction protocol.

Several limitations need however to be acknowledged: first, the majority of patients who underwent this protocol had only a mild allergic reaction after the first dose. Thus, this protocol may not be tolerated by vaccine-sensitized patients with severe allergic reaction after the first dose. Secondly, we have not validated the specificity of the skin testing nor the BAT with a formal drug challenge. More data are to come with ongoing clinical trials evaluating the safety of administering a second dose of COVID-19 mRNA vaccines in individuals who experienced systemic allergic reactions to the first vaccine injection (NCT04977479). Third, we unexpectedly observed a significantly increased number of vaccine-sensitized patients in individuals who received the mRNA-1273 vaccine, although this vaccine is not associated with a higher prevalence of allergic reaction as compared to the BNT162b2 mRNA vaccine. Importantly, these results were confirmed by BAT and were specific to the post-vaccination cohort, since $53 \%$ of individuals with negative skin tests in the pre-vaccination cohort were screened with the mRNA-1273 vaccine and not with the BNT162b2 mRNA. To our opinion, urgent studies are warranted to better understand the mechanisms and potential clinical consequences of vaccine-induced sensitization.

In conclusion, we demonstrated that IDT and BAT with mRNA-based vaccines can be useful to evaluate patients with allergic-like reactions after the first injection with an mRNA vaccine and decide which vaccination protocol to use for the booster dose. Ultimately, with few exceptions, allergies, if properly investigated, should not be any more a contra-indication to vaccination in this deadly pandemic.

Author contribution: Conceptualization and initiation of the study: YDM. Supervision: YDM. Formal analysis: FS and YDM. Contribution to the design of the study: DY, FS, TH, AM, CG, SM and CR. Collection of the data: FS, RMA, LC, VMB. BAT analysis: DY and KK. Writing-original draft: FS and YDM. Writing-review \& editing: TH and CR. 
Acknowledgment : we thank Prof. Pantaleo, Prof Genton and Prof Calandra for their support in the conduction of this study. We thank Alba Santos, Lucy Kunz, Chiara Marinoni and Patrick Genoud for their help in reporting suspect allergic reactions.

Conflict of interests : DY and KK are employees of ADR-AC GmbH (Switzerland). The authors have no other financial disclosure.

ORCID numbers: Yannick Muller 0000-0003-0513-7156 . Camillo Ribi0000-0002-0446-9741. Daniel Yerly0000-0003-2857-0737. Alix Miauton0000-0003-1849-8874. Sylvain Meylan0000-0001-6319-2423.

\section{References}

1. Cabanillas B, Akdis C, Novak N; Allergic reactions to the first COVID-19 vaccine: a potential role of Polyethylene glycol. Allergy 2020;

2. Shimabukuro TT, Cole M, Su JR; Reports of Anaphylaxis After Receipt of mRNA COVID-19 Vaccines in the US-December 14, 2020-January 18, 2021. JAMA 2021;

3. Shimabukuro T, Nair N; Allergic Reactions Including Anaphylaxis After Receipt of the First Dose of Pfizer-BioNTech COVID-19 Vaccine. JAMA 2021;

4. Stone CA, Liu Y, Relling MV et al.; Immediate Hypersensitivity to Polyethylene Glycols and Polysorbates: More Common Than We Have Recognized. J Allergy Clin Immunol Pract 2019; 7: 1533.

5. Shavit R, Maoz-Segal R, Iancovici-Kidon M et al.; Prevalence of Allergic Reactions After Pfizer-BioNTech COVID-19 Vaccination Among Adults With High Allergy Risk. JAMA Netw Open 2021; 4: e2122255.

6. Klein NP, Lewis N, Goddard K et al.; Surveillance for Adverse Events After COVID-19 mRNA Vaccination. JAMA 2021;

7. Greenhawt M, Abrams EM, Shaker M et al.; The Risk of Allergic Reaction to SARS-CoV-2 Vaccines and Recommended Evaluation and Management: A Systematic Review, Meta-Analysis, GRADE Assessment, and International Consensus Approach. J Allergy Clin Immunol Pract 2021; S2213.

8. Pitlick MM, Sitek AN, Kinate SA et al.; Polyethylene glycol and polysorbate skin testing in the evaluation of coronavirus disease 2019 vaccine reactions: Early report. Ann Allergy Asthma Immunol 2021; 126: 735.

9. Sellaturay P, Nasser S, Islam S et al.; Polyethylene glycol (PEG) is a cause of anaphylaxis to the Pfizer/BioNTech mRNA COVID-19 vaccine. Clin Exp Allergy 2021; 51: 861.

10. Sellaturay P, Nasser S, Ewan P; Polyethylene Glycol-Induced Systemic Allergic Reactions (Anaphylaxis). J Allergy Clin Immunol Pract 2021; 9: 670.

11. Muraro A, Roberts G, Worm M et al.; Anaphylaxis: guidelines from the European Academy of Allergy and Clinical Immunology. Allergy 2014; 69: 1026.

12. Muraro A, Agache I, Clark A et al.; EAACI food allergy and anaphylaxis guidelines: managing patients with food allergy in the community. Allergy 2014; 69: 1046.

13. Muraro A, Worm M, Alviani C et al.; EAACI guideline: Anaphylaxis (2021 update). Allergy 2021;

14. Smiljkovic D, Blatt K, Stefanzl G et al.; BTK inhibition is a potent approach to block IgE-mediated histamine release in human basophils. Allergy 2017; 72: 1666.

15. Wood RA, Berger M, Dreskin SC et al.; An algorithm for treatment of patients with hypersensitivity reactions after vaccines. Pediatrics 2008; 122: e771.

16. Hourihane JO, Byrne AM, Blümchen K et al.; Ascertainment Bias in Anaphylaxis Safety Data of COVID-19 Vaccines. J Allergy Clin Immunol Pract 2021; 9: 2562. 
17. Blumenthal KG, Robinson LB, Camargo CA et al.; Acute Allergic Reactions to mRNA COVID-19 Vaccines. JAMA 2021; 325: 1562.

18. Zhou ZH, Stone CA, Jakubovic B et al.; Anti-PEG IgE in anaphylaxis associated with polyethylene glycol. J Allergy Clin Immunol Pract 2020;

19. Bruusgaard-Mouritsen MA, Jensen BM, Poulsen LK et al.; Optimizing investigation of suspected allergy to polyethylene glycols. J Allergy Clin Immunol 2021; S0091.

20. Jover Cerdá V, Rodríguez Pacheco R, Doménech Witek J et al.; Immediate hypersensitivity to polyethylene glycols in unrelated products: when standardization in the nomenclature of the components of drugs, cosmetics, and food becomes necessary. Allergy Asthma Clin Immunol 2019; 15: 9.

21. Banerji A, Wickner PG, Saff R et al.; mRNA Vaccines to Prevent COVID-19 Disease and Reported Allergic Reactions: Current Evidence and Suggested Approach. J Allergy Clin Immunol Pract 2020;

22. Wenande E, Garvey LH; Immediate-type hypersensitivity to polyethylene glycols: a review. Clin Exp Allergy 2016; 46: 907.

23. Hyry H, Vuorio A, Varjonen E et al.; Two cases of anaphylaxis to macrogol 6000 after ingestion of drug tablets. Allergy 2006; 61: 1021.

24. Bommarito L, Mietta S, Nebiolo F et al.; Macrogol hypersensitivity in multiple drug allergy. Ann Allergy Asthma Immunol 2011; 107: 542.

25. Sohy C, Vandenplas O, Sibille Y; Usefulness of oral macrogol challenge in anaphylaxis after intra-articular injection of corticosteroid preparation. Allergy 2008; 63: 478.

26. Kelso JM; Misdiagnosis of systemic allergic reactions to mRNA COVID-19 vaccines. Ann Allergy Asthma Immunol 2021; 127: 133.

27. Troelnikov A, Perkins G, Yuson C et al.; Basophil reactivity to BNT162b2 is mediated by PEGylated lipid nanoparticles in patients with PEG allergy. J Allergy Clin Immunol 2021; 148: 91.

28. Warren CM, Snow TT, Lee AS et al.; Assessment of Allergic and Anaphylactic Reactions to mRNA COVID-19 Vaccines With Confirmatory Testing in a US Regional Health System. JAMA Netw Open 2021; 4: e2125524.

29. Kozma GT, Mészáros T, Vashegyi I et al.; Pseudo-anaphylaxis to Polyethylene Glycol (PEG)-Coated Liposomes: Roles of Anti-PEG IgM and Complement Activation in a Porcine Model of Human Infusion Reactions. ACS Nano 2019; 13: 9315.

30. Sahin U, Muik A, Vogler I et al.; BNT162b2 vaccine induces neutralizing antibodies and poly-specific T cells in humans. Nature 2021; 595: 572.

31. Fenwick C, Turelli P, Pellaton C et al.; A high-throughput cell- and virus-free assay shows reduced neutralization of SARS-CoV-2 variants by COVID-19 convalescent plasma. Sci Transl Med 2021; 13: eabi8452.

\section{Figures/Tables}

\section{Figure 1: Skin testing with COVID-19 mRNA vaccines}

(A) Representative intradermal (IDR) skin tests results of a patient with history laxative anaphylaxis. The picture was taken 20 minutes after IDR for the BNT162b2 mRNA and mRNA-1273 vaccines (1/100), PEG 2000 1\% (1/100), Trometamol 1\% (1/100). All dilutions were performed in $\mathrm{NaCl} \mathrm{0.9 \% .} \mathrm{(B)} \mathrm{Flow} \mathrm{chart} \mathrm{of}$ individuals from the pre-vaccination and post vaccination cohorts, which were evaluated in the division of allergy at the university hospital of Lausanne. Abbreviation: Polyethylene glycol (PEG), Trometamol (TRIS), Intradermal reaction (IDR).

Figure 2: Basophil activation tests (BAT) 
A-C) Percentage of CD63 and CD203c expression after gating on CCR3+ basophils. 27 BATs were interpreted as positive and 17 as negative for a sensitization to COVID-19 mRNA vaccines. (A) Peripheral blood was incubated with IL-3 and different concentration of COVID-19 mRNA vaccines or polysorbate-80. Anti-IgE antibodies were used as positive control and medium-only) as negative control. Percentage of CD63 expression is shown. (B) Peripheral blood was incubated with different concentration of COVID-19 mRNA vaccines or polysorbate-80 without IL-3. CD63 expression is shown. (C) Peripheral blood was incubated with different concentration of COVID-19 mRNA vaccines or polysorbate-80. CD203c expression is shown. (A-C) AntiIgE antibodies were used as positive control and medium-only as negative control. (D) Correlation of BAT results with skin test results (when available). Multiple unpaired T test was performed. Mean and standard deviation are shown. ${ }^{*} \mathrm{P}<0.05$. Abbreviations: Negative (Neg), Positive (Pos).

Figure 3: Ibrutinib and basophil activation tests (BAT)

Percentage of CD63 (+/- IL3) and CD203c expression after gating on CCR3+ basophils in five individuals which were sensitized to the COVID-19 mRNA vaccines. 100nM of Ibrutinib was added to control condition (medium-only), basophils were activated with anti-IgE monoclonal antibodies, or fMLP, or $0.1 \%$ of the BNT162b2 mRNA or mRNA-1273 vaccines. Multiple unpaired T test was performed. Mean and standard deviation are shown. ${ }^{*} \mathrm{P}<0.05,{ }^{* *} \mathrm{P}<0.01,{ }^{* * *} \mathrm{P}<0.001$.

\section{Table 1}

Characteristics of the 87 patients tested for the mRNA vaccines after experiencing an allergy-like reaction immediately after immunization. Fisher's exact tests were performed.

* including inconclusive intradermal skin tests

\section{Table 2}

Characteristics of sensitized patients who underwent a five-step induction protocol with either the BNT162b2 mRNA or the mRNA-1273 vaccines. Abbreviations: Not done (ND), induction tolerance (IT), Intradermal reaction $(\mathrm{IDR})$

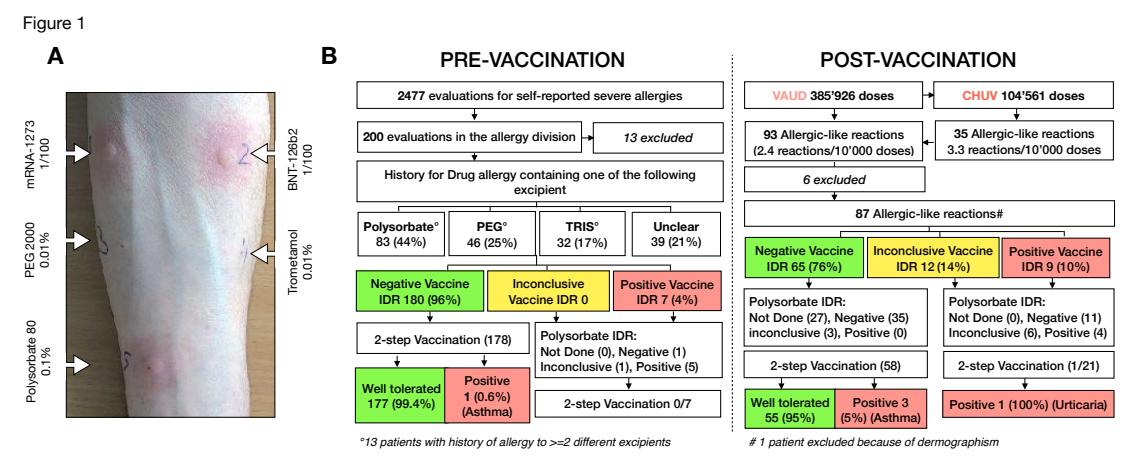



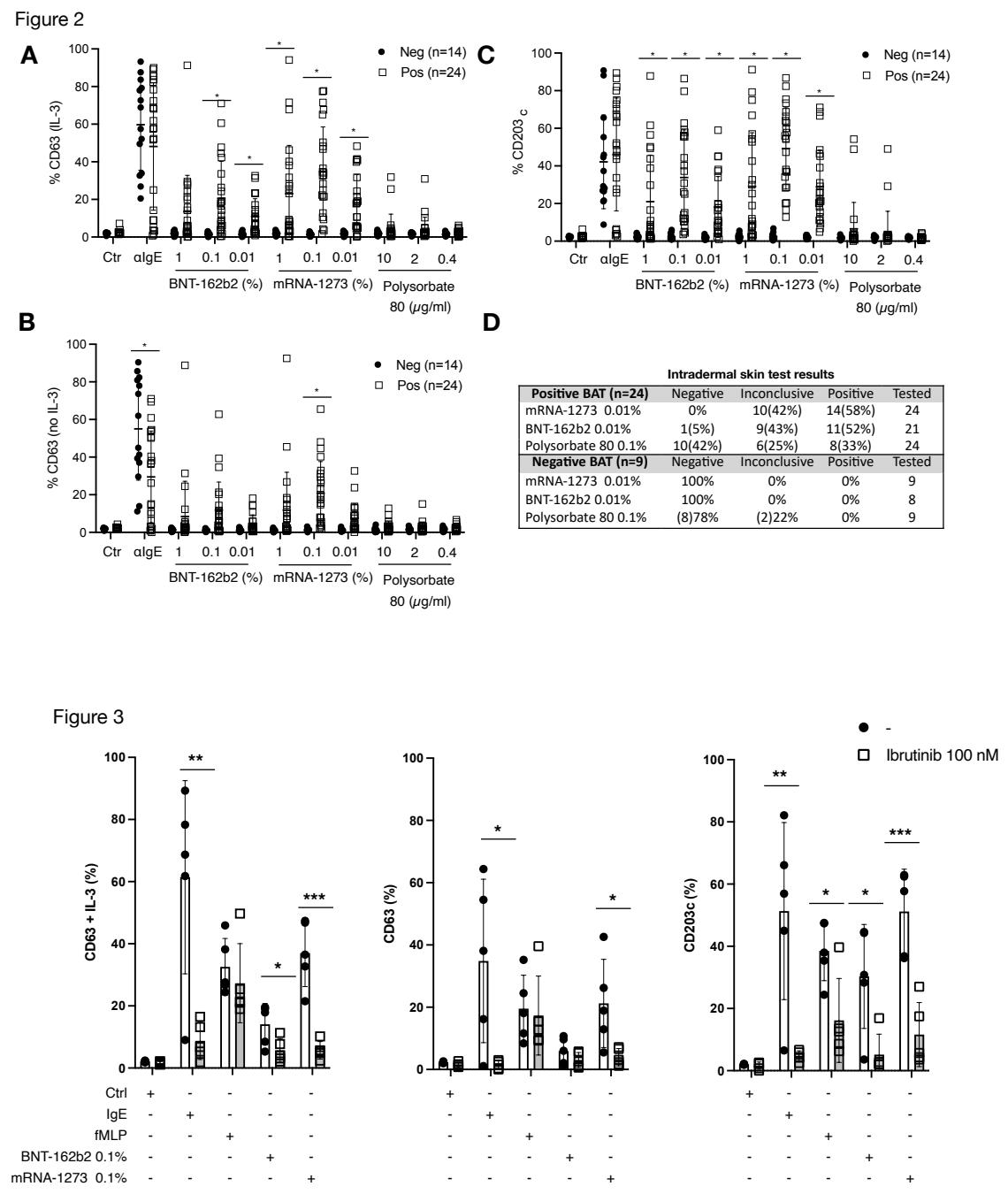
Table 1

\begin{tabular}{|c|c|c|c|}
\hline & Skin Tests Neg & Skin Tests Pos* & $p$ value \\
\hline Total of patients & 65 & 21 & \\
\hline Age (mean +/-SD)) & $51.2(+/-15.1)$ & $40.8(+/-15.2)$ & $<0.01$ \\
\hline Female & $57(88 \%)$ & $20(95 \%)$ & ns \\
\hline BNT-162b2 (\%) & $43(66 \%)$ & $3(14 \%)$ & $<0.01$ \\
\hline Prior allergy sharing additives with vaccines & $2(3 \%)$ & $1(5 \%)$ & ns \\
\hline Prior anaphylaxis & $27(41.5 \%)$ & $7(33 \%)$ & $n s$ \\
\hline Prior allergy (any) & $60(92 \%)$ & $12(57 \%)$ & $<0.01$ \\
\hline Normal Basal Tryptase & 50/51 (98\%) & 19/19 (100\%) & $n s$ \\
\hline Symptoms & & & $n s$ \\
\hline Cutaneous & $53(82 \%)$ & $17(81 \%)$ & \\
\hline Respiratory & $22(33 \%)$ & $6(29 \%)$ & \\
\hline $\begin{array}{l}\text { Digestive } \\
\text { Dige }\end{array}$ & $7(11 \%)$ & $1(5 \%)$ & \\
\hline Cardiovascular & $2(3 \%)$ & $1(5 \%)$ & \\
\hline Treatment & & & ns \\
\hline AntiH1, Steroids only & $50(77 \%)$ & 17 (81\%) & \\
\hline Autoresolutive & $12(19 \%)$ & $4(19 \%)$ & \\
\hline Epinephrine & $3(5 \%)$ & 0 & \\
\hline Timing & & & ns \\
\hline Timing $\leq 30 \mathrm{~min}$ & $42(65 \%)$ & $11(52 \%)$ & \\
\hline Timing 30-60min & $2(3 \%)$ & 0 & \\
\hline Timing $>60 \mathrm{~min}$ & $21(32 \%)$ & $10(48 \%)$ & \\
\hline Anaphylaxis EAACl criteria & $16(25 \%)$ & $2(10 \%)$ & $n s$ \\
\hline Brighton Scale & & & ns \\
\hline I & $5(8 \%)$ & $2(10 \%)$ & \\
\hline II & $9(14 \%)$ & 0 & \\
\hline & $1(2 \%)$ & 0 & \\
\hline No criteria & $50(77 \%)$ & 19 (91\%) & \\
\hline Ring and Messmer Severity Scale & & & ns \\
\hline 1 & $40(62 \%)$ & $13(62 \%)$ & \\
\hline II & $18(28 \%)$ & $8(38 \%)$ & \\
\hline III & $7(11 \%)$ & 0 & \\
\hline IV & 0 & 0 & \\
\hline
\end{tabular}

\begin{tabular}{|c|c|c|c|c|c|c|c|c|c|c|c|c|c|c|c|}
\hline $\mathrm{Age}$ Sex & ex Cohor & $\begin{array}{c}\text { Timing of } \\
\text { the reaction }\end{array}$ & 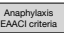 & 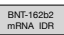 & $\begin{array}{l}\text { MANA-1273 } \\
\text { IDR }\end{array}$ & 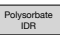 & 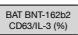 & 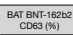 & 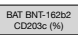 & 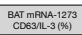 & $\begin{array}{l}\text { BAT MRNA-1273 } \\
\text { CDES(80) }\end{array}$ & $\begin{array}{l}\text { BAT meNA-1273 } \\
\text { CO20200 (15\%) }\end{array}$ & Vacocine used & Completed & Reaction during II \\
\hline $75 \mathrm{~F}$ & $F \quad$ post & $>60 \mathrm{~min}$ & No & inconclusive & inononlusve & $\begin{array}{ll}\text { negative } \\
\end{array}$ & ${ }_{4}^{4}$ & 3.4 & 3.6 & 5,8 & 8 & 38,1 & MANA-1273 & Yes & \\
\hline $\begin{array}{ll}22 & F \\
41 & F\end{array}$ & $\begin{array}{ll}F & \text { post } \\
F & \text { post }\end{array}$ & $\begin{array}{l}306 \mathrm{~min} \\
=30 \mathrm{~min} \\
5\end{array}$ & $\begin{array}{l}\text { No } \\
\text { No }\end{array}$ & $\begin{array}{l}\text { posstive } \\
\text { postive }\end{array}$ & $\begin{array}{l}\text { posaltive } \\
\text { postrve }\end{array}$ & $\begin{array}{l}\text { posalive } \\
\text { postive }\end{array}$ & $\begin{array}{l}4,7 \\
\text { No } \\
\text { No }\end{array}$ & $\begin{array}{l}2,3 \\
\text { No } \\
\text { No }\end{array}$ & $\begin{array}{l}5,9 \\
\text { No }\end{array}$ & $\begin{array}{l}1,8 \\
\text { No }\end{array}$ & $\begin{array}{l}44,6 \\
\text { No }\end{array}$ & $\begin{array}{l}68.8 \\
\text { No }\end{array}$ & 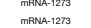 & $\begin{array}{l}\text { Yess } \\
\text { Yes }\end{array}$ & $\cdot$ \\
\hline $37 \quad \mathrm{~F}$ & $F$ post & $>60 \mathrm{~min}$ & № & No & inonolusisve & posalive & 0,4 & 0,8 & 15,1 & 0,1 & 2 & 28,3 & meNA-1273 & Yes & . \\
\hline $46 \mathrm{~F}$ & $F$ post & $260 \mathrm{~min}$ & No & inconcluswe & inconcluswe & negative & 60.5 & 39,3 & 79.7 & 48,3 & 23,4 & 54,2 & meNA-1273 & Yes & \\
\hline $53 \mathrm{~F}$ & $F$ post & =30 min & № & No & posatve & inconoluswe & 14,3 & 6,3 & 25,1 & 10,7 & 7.8 & 20,1 & menN-1273 & Yes & \\
\hline $36 \mathrm{~F}$ & $F$ post & $=30 \mathrm{~min}$ & No & inconcluswe & postitue & negative & 60,5 & 39,3 & 79,7 & 48,3 & 23,4 & 54,2 & mPNA-1273 & Yes & Local unticaria \\
\hline $52 \quad \mathrm{~F}$ & $F$ post & $>660 \mathrm{~min}$ & No & inconecluswe & inonoclusive & negative & 11,5 & 9,1 & 10.4 & 41.2 & 40.2 & 37 & mANA-1273 & Yes & \\
\hline $52 \quad \mathrm{~F}$ & $F$ post & $=30 \mathrm{~min}$ & Yes & positive & postive & negative & 33.6 & 34.6 & 55,5 & 24,4 & 29,4 & 47,3 & mPNA-1273 & Yes & \\
\hline $39 \mathrm{~F}$ & & $=30 \mathrm{~min}$ & № & No & postive & postive & 26,4 & 14,3 & 40,2 & 18 & 27,7 & 60 & meNA-1273 & Yes & . \\
\hline $\mid \begin{array}{ll}66 & F \\
45 & F\end{array}$ & F $\begin{array}{ll}\text { fore } \\
\text { F }\end{array}$ & & & $\begin{array}{l}\substack{\text { postive } \\
\text { neagative }} \\
\text { nat }\end{array}$ & postive & Inconclusvive & 16,6 & 2,8 & 37,2 & 19,1 & 8 & 54,7 & BNT-162022 m PANA & Yess & \\
\hline & & & & negative & negative & negative & No & No & & No & & & ENT-16262 mPNA & Yes & $\begin{array}{l}\text { Mild dasthantic } \\
\text { exacerbation }\end{array}$ \\
\hline $66 \quad \mathrm{~F}$ & $F$ pre & & & postive & postive & negative & 5,3 & 1,2 & 3,6 & 5,1 & 5.5 & 36,8 & BNT-16202 m mNA & Yes & 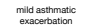 \\
\hline $\begin{array}{ll}68 & F \\
\end{array}$ & F pre & - & - & positive & postive & positive & 0.5 & 0.4 & 4.4 & 1.3 & 2,2 & 12,8 & BNT-162b2 mPNA & Yes & \\
\hline
\end{tabular}

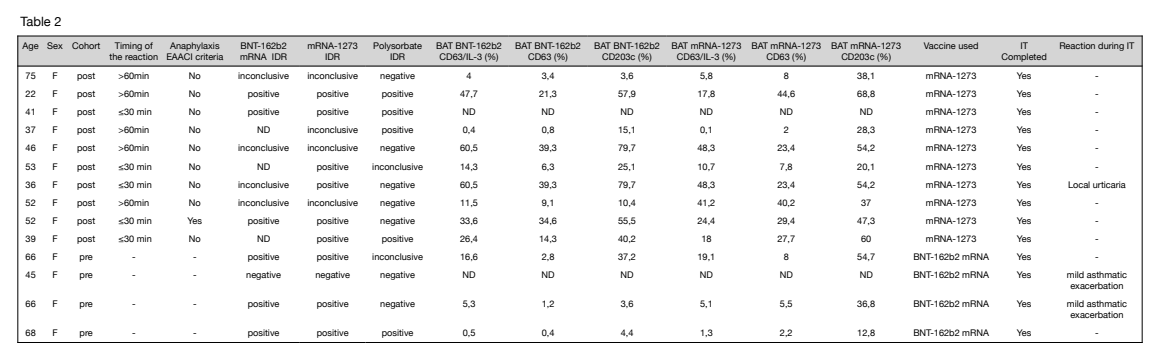

